\title{
Event-Related Brain Potentials and Case Information in Syntactic Ambiguities
}

\author{
Jens-Max Hopf, Josef Bayer, Markus Bader, and Michael Meng
}

Friedrich-Schiller-Universität Jena. Germany

\begin{abstract}
an anP study German sentences were investigated that contain a case-ambiguous NP that may be assigned accusative or dative case. Sentences were disambiguated by the verb in final position of the sentence. As our data show. sentences ending in a verb that assigns dative case to the ambiguous NP elicit a clear garden-path effect. The garden-path effect was indicated by a broad centro-posterior negative shift that occurred between 300 and $900 \mathrm{msec}$ after the dative-assigning verb was presented. No enhanced $\mathrm{P} 600$ following the misanalysis was observed. Noun phrases whose case ambiguity was resolved in favor of accusative case and unambiguously dativemarked NPs did not trigger significant ERP differences. We will discuss the implications of our results for parsing and its
\end{abstract}

\section{INTRODUCTION}

\section{Parsing and Garden-Path Sentences}

In order to successfully understand spoken or written language, different types of information. linguistic as well as nonlinguistic. have to be combined to derive the message that an utterance encodes. One of the great challenges in the study of the human sentence processing mechanism (HSPM) is to find out how different types of information are used on-line. From the perspective of processing. there is a question about the point at which. in the comprehension process, specific pieces of information come into play and how they constrain each other. With respect to the neuropsychological correlates of processing, this relates to the problem of whether the employment of different information types can also be distinguished by virtue of the brain's responses.

To study the time course of processing, much research has been aimed at specifying how the HSPM copes with the problem of ambiguity (i.e., the fact that the input is often not sufficiently rich to be assigned a unique representation). Ambiguities may arise at all levels of the comprehension process, but research into ambiguity resolution has proven to be especially fruitful in the area of parsing. Parsing refers to the process during which a syntactic representation is assigned to an input string of words. The special interest that ambiguities have received in parsing is due to the fact that temporary neuropsychological correlates. The results of this study support a parser design according to which the s()-called structural case (nominative or accusative) is assigned without any delay in the absence of morpho-lexical counterevidence. It is argued that the enhancement of a negative ERP component with a "classical" N400 topography reflects the difficulty of reanalysis due to reaccessing morpho-lexical information that lies outside the domain of the parsing module. Consequently. ERP responses to garden-path effects are not confined to a late positivity but vary depending on the level of processing involved in reanalysis The fact that garden-path effects may also elicit an N400 can be linked to the nonhomogeneous linguistic properties of the constructions from which ther arise. 
bution into the prepositional phrase (PP) as in (2b) and neglects the attachment possibility that is required in (2a).

In serial models of parsing. the preferred structure is the only structure that the parser actually computes. with all alternatives being discarded (Frazier, 1978: Frazier \& Rayner 1982). Parallel models that hold that the parser computes all alternatives available usually invoke the concept of ranking to explain the processing asymmetries: The preferred structure is ranked highest and thus is most accessible to the parser (Gibson, 1991: Gorrell, 198-). Finally delay models claim that at a point of ambiguity the parser tries to postpone any commitment (look-ahead. see Marcus. 1980) or that it only builds an underspecified structure (Weinberg, 1993). But garden-path effects dictate that look-ahead must be limited and that even partial commitments must be specific enough to rule out those structures that cause processing difficulties when the sentence gets disambiguated. Given the general approach just outlined, garden-path effects will arise if the preferred structural assignment turns out to be incorrect at the point of disambiguation and revisions subsequently become necessary. Reanalysis of an initial structural assignment is responsible for the perceived processing difficulty:

One important aspect in parsing research is to specify the criterion according to which the parser selects the preferred structure. Again, although the models differ with respect to the details of implementation. they almost uniformly assume that the parser will assign the minimal structure that is compatible with the input requirements, a property that possibly derives from the interaction of time constraints and capacity limitations. This point is important because it presupposes that preferences hinge upon the existence of structural differences among alternative continuations. Research into the process of reanalysis constitutes another cornerstone of parsing theory. which has been the focus of many recent studies. Revisions at the level of syntactic representation have usually been considered central to the explanation of garden-path effects, but one question is whether revisions at other levels of representation (e.g.. the lexicon) may give rise to processing difficulties as well. To successfully approach this question we need a technique that is able to distinguish processing effects at different representational levels.

\section{Garden Path Sentences and Event-Related Potentials}

One such promising technique is the study of eventrelated brain potentials (ERPs). ERPs provide a very close link $\mathrm{t}(\mathrm{)}$ the brain functionality involved in language comprehension. Compared to standard on-line techniques. ERPs have various advantages that make them an interesting tool tor the study of language processing (see Kutas \& Van Petten. 1994: Osterhout. 1994 for a comprehensive discussion). Most important for our purposes is the fact that the phasic nature of cortical potentia. changes provides a unique opportunity to disentangle fast and sometimes hidden cognitive processes that $x$. cur at different levels of processing without confound. ing them with the preparation and execution of over: responses.

Kutas and Hillyard (1980) were the first to show reliable variations of an ERP component (N400) to the final word of a sentence that violates semantic constraints of the sentence. This $\mathrm{N} 400$ a negative-going com ponent in a time range of about 250 to $600 \mathrm{msec}$ after word onset, turned out to be also elicited by semantically correct but unusual words (Kutas \& Hillyard, 1984). Therefore. N400 was assumed to correspond to the degree of semantic priming of a word within the prevailing context (Van Petten \& Kutas, 1991).

The processing of ungrammatical sentences has also been investigated in a number of studies. For example. subcategorization ( $3 \mathrm{a}$, below) and agreement violations (3b), ungrammatical wh-questions ( $3 c$ ) , and violations of conditions on phrase structure ( $3 \mathrm{~d}$ ) are associated with a characteristic late positive-going component that has a broad topographical distribution and starts about 500 msec after the word (italicized in 3) that indicates the mismatch (Neville. Nicol. Barss. Forster. \& Garrett, 1991: Osterhout \& Holcomb, 1992: Osterhout, Holcomb. \& Swinney. 1994; Osterhout \& Mobley: 1995).

(3) a. "The woman persuaded to answer the door

b. ${ }^{*}$ The elected officials bopes to succeed.

c. ${ }^{*}$ What ${ }_{i}$ was $\left[\mathrm{NP}\right.$ a $\left[\mathrm{x}\right.$ picture of $\left.\left.\mathrm{t}_{\mathrm{i}}\right]\right]$ [vp printed by the newspaper]?

d. "The man admired Don's of sketch the landscape.

This positivity is known as the $\mathrm{P} 600$, or syntactic positive shift (SPS) (Hagoort. Brown. \& Groothusen, 1993; Osterhout \& Holcomb. 1992). In phrase structure violations. the late positivity is preceded by an enhanced left anterior negativity (N125. Neville et al., 1991: N180. Friederici. Pfeifer. \& Hahne. 1993) and a strongly left-lateralized negativity around $400 \mathrm{msec}$ (Neville et al.. 1991). In sum. the perception of syntactic anomalies is not associated with a component as distinct as the N400. Instead, the brain's response apparently varies depending on the actual type of syntactic riolation that is involved. Most importantly; however, is the fact that the ERP variations to syntactic anomalies differ from those that are obtained in the area of lexico-semantic processing. Therefore. ERPs seem to be sensitive to different levels of processing

Although sentences that contain a semantic anomals or are downright ungrammatical have already received a good deal of attention. only a few studies have invest: gated the processing of garden-path sentences. A thr-i investigation of ERP responses to garden-path sentencihas been carried out by Osterhout and Holcomb (190? This study looked at the processing of simple actro 
sentences as in (4) vs. reduced relative clauses as in (5). which would only be legitimate as a noun phrase (XP):

(4) The broker hoped to sell the stock ("was sent to) jail)

(5) The broker persuaded to sell the stock (was sent to jail)

Because the parser initially computes a simple active sentence. the word to in (5) seems to violate the subcategorization requirement of the transitive verb persuade. Perception of to evoked a characteristic late positive-going component that occurred about $500 \mathrm{msec}$ after this word with a broad right fronto-central distribution (P600). In another study, Osterhout et al. (1994) presented sentences in which. due to the missing complementizer that. the postverbal NP is locally ambiguous between a direct object and the subject of a clausal complement, as in (6).

(6) The lawyer charged (that) the defendant was lying.

Again. a positive deflection between 500 and $800 \mathrm{msec}$ after the auxiliary in (6) indicated that in initially pursuing a direct-object analysis of the matrix clause. subjects were garden-pathed upon the perception of the disambiguating auxiliary: Evidence for late positivities in syntactic garden-path constructions was also reported in Friederici and Mecklinger (1996). A positivity of similar distribution but with an earlier onset and peak latency (P345) was found by Mecklinger. Schriefers. Steinhauer. and Friederici (1995) in response to garden-path sentences that enforce the revision of an initial filler gap assignment.

So far, the cognitive events underlying late positivities like the P600/SPS are not sufficiently understood. Osterhout et al. (1994) cautiously suggest that the P600 might be tied to the "cost of reprocessing following the garden path." (i.e., reanalysis: cf. Friederici. 1995. for a similar hypothesis). This raises the question of whether positivities like the P600/SPS are a general indictor of reanalysis. or whether they are specific to revisions at the phrase structure level of representation. Note, however. that the P600/SPS has also been found in structures that remain ungrammatical under any analysis. Therefore, positivities like the P600/SPS might indicate the detection of a (temporary) ungrammaticality that is characteristic for both the perception of garden-path sentences and sentences that are irreversibly ungrammatical.

As a first step toward an answer we need to know the brain's response to garden-path effects that do not involve any change at the level of phrase structure but involve changes at a different level of representation. In this study we will report experimental results of the processing of a novel type of ambiguity-pure case ambiguity-that we think provide relevant data. Pure case ambiguities (PCAs) may lead to garden-path effects that do not necessitate changes at the level of phrase structure representation. Before we outline the processing issues and the experimental data, we will introduce the notion of PCA and present the necessary linguistic background

\section{Pure Case Ambiguities}

Enlike English. where the syntactic function of a phrase is largely determined by the order of constituents. German allows much more freedom with respect to the order of syntactic constituents. The identification of the syntactic function of an NP is to a large extent dependent on its case morpholog: In principle. determiners. prenominal adjectives. and nouns are inflected for case. At the same time. however. the case paradigms of the modern language are everything but perfect. Especially with respect to nouns. we find much advanced case syncretism (i.e.. morphological nondistinctness in the paradigm). There are four cases in German (nominative. genitive dative, and accusative). Bare NPs like proper names (Maria) mav be three-wars ambiguous and certain indefinite plurals (Studenten: students: Dirigenten: conductors) may even be four-ways ambiguous. Given the important role that case information plays due to the word-order freedom of German. case ambiguities may easily lead to syntactic function ambiguities that in turn fall into two groups: (1) subject-object ambiguities and (2) object-object ambiguities. Whereas subject-object ambiguities are often characterized by alternative fillergap dependencies (Bader. 1994a: Bader. 1994b: Hemforth. 1993). object-object ambiguities are based on identical phrase structural representations. Consider the examples in Table 1.

In Table 1. (7), the case-ambiguous NP Studenten may function either as an accusative object $\left({ }^{\top}\right.$ a) or as a dative object ( $7 \mathrm{~b})$. The sentences are disambiguated by the clause final main verbs: The verb seben (to see) assigns accusative case to its object. whereas the verb belfen (to help) assigns dative case. Because these alternative assignments are not mirrored by alternative phrase markers. we dub them PCAs. ${ }^{1}$ According to native speaker intuitions, sentences such as ( $/ a)$ and (-b) are parsed without any noticeable difficulty. This. however. does not seem to be the case if the distance between the ambiguous NP and the disambiguating verb is increased as in (8). The two sentences in (8) reflect the same objectobject ambiguity as those in ( $)$ ): The verb umjubeln (to cheer) requires an accusative object. whereas applaudieren (to applaud) requires a dative object. Both sentences are perfectly grammatical. If. however, the initial ambiguous NP must be interpreted as a dative object. as in (8b). informants often report processing difficulties at the final verb of the sentence. Contrary to this. (8a), where the bare $\mathrm{NP}$ has an assigned accusative case. is processed easily. In addition. sentences like ( $8 \mathrm{c}$ ). in which the bare $\mathrm{NP}$ is unambiguously inflected for dative case (dative Musikern vs. nominative/accusative Musiker), do not invoke processing difficulties. 
Table 1. Sentence examples. Examples ( $8 a-c)$ show one triple of accusative ambiguous ( $A A$ ), dative ambiguous (DA), and dative unambiguous (DL) sentences used in the questionnaire study and the ERP experiment.

\begin{tabular}{ll}
\hline (7a) & Vielleicht bat er Studenten gesebenacc. \\
accusative & perhaps has he students seen. \\
ambiguous & Vielleicht hat er Studenten gebolfen dat \\
(7b) & perhaps has he students helped. \\
dative &
\end{tabular}

(8a)

accusative ambiguous (AA)

(8b)

\section{dative} ambiguous (DA)

\section{(8c)}

\begin{tabular}{|c|c|}
\hline $\begin{array}{l}\text { dative } \\
\text { unambiguous }\end{array}$ & $\begin{array}{l}\text { musicians who a difficult opus rehearsed } \\
\text { have }\end{array}$ \\
\hline$(D C)$ & $\begin{array}{l}\text { kann ein Kritiker rubig } \\
\text { applaudieren } \\
\text { dir. }\end{array}$ \\
\hline
\end{tabular}

Intuitive judgments on the relative processing difficulty of PCAs suggest that PCAs may give rise to garden-path effects. This suggests that case ambiguities are resolved immediately; in analogy to more familiar types of ambiguities (phrase structure and filler-gap amhiguities). We hypothesize that the initial noun in sentences like ( $8 \mathrm{a} \& \mathrm{~b}$ ) is assigned the accusative case before the case-assigning verb in the final position of the clause is encountered and that the information that this noun could equally well carry the dative case is lost at this point. If the verb turns out to assign the dative case. a processing difficulty is perceived. Secondly, given our structural assumptions, the processing effect must be tied directly to the morphological ambiguity of the bare NP hecause reanalysis after the garden path in sentences like ( 8 b) does not involve phrase structure revisions and there is no garden-path effect in sentences with unambiguously intlected nouns as in (8c).

If garden-path effects can be demonstrated experi- mentally in the processing of PCAs. interesting questions arise both with respect to parsing and its neurophysiological manifestations. From a parsing perspective, the question arises which principles are used to resolve case ambiguities on-line. Because the choice of dative or accusative case has no phrase structural consequences. none of the economy-driven principles that have been proposed in the area of syntactic processing would predict a difference. Furthermore. a principled account of the reanalysis operations that become necessary at the point of disambiguation has to be given, which allows the evaluation of processing effects in the absence of phrase structure revisions

Although parsing issues will be discussed in this paper, we will concentrate on the neuropsychological perspective. The main question is what electrocortical signs are characteristic for the garden-path effect in PCAs. As we have indicated above, garden-path effects at the syntactic level are tied to late positivities. but there is an ongoing debate concerning the proper functional interpretation of those components. Electrocortical signs in resporise to the garden-path effect produced by PCAs may significantly contribute to that debate. As with garden-path effects in general, readers perceive a temporary ungrammaticality if the continuation they choose at the point of ambiguity turns out to be contradicted by disambiguating input. In PCAs, readers perceive a case mismatch if they encounter a dative-assigning verb because what they actually expect is a verb that assigns the accusative case. However, the necessary revision does not affect the phrase structural representation of the sentence but rather the case features of the ambiguous NP. If late positivities are tied to the perception of a syntactic anomaly or reanalysis operations in general, we should expect them to occur with PCAs as well. If, on the other hand. those components are more specifically linked to processes of phrase-structural revisions, they are unlikely to occur in the present context.

\section{RESULTS}

The results of the ERP-study are preceded by those derived from a questionnaire study that served as the base line for the actual ERP investigation.

The ERP data focus on positions of ambiguity and on ambiguity resolution. ERPs to relative clauses will not be analyzed because their in-toto presentation on the video screen gave rise to massive eve movement artifacts. For the sake of convenience. the following abbreviations will be used: ST for sentence type. ES for electrode site. DA for dative ambiguous. $D C$ for dative unambiguous. and At for accusative ambiguous. Multivariate analysis of variance (ILANOVA) I refers to overall (ST and ES) MAYOVAs based on the raw data, and MANOVA II is based on direction cosines of the ST vectors to evaluate true interactions between ST and ES (see "Data Analysis") 


\section{The Questionnaire Study}

The purpose of this experiment was to confirm the intuitively felt processing difficulty of DA sentences such as (8b) in contrast to AA sentences like (8a) and $\mathrm{DC}$ sentences like $(8 \mathrm{c})$. Furthermore, sentences were pretested to be used in the later ERP experiment. Table 2 shows the overall percentage of "awkward" responses for the two sets in which the materials (including filler sentences) were organized. as well as the results collapsed over both sets (see "Methods" section). Responses to sentences that were finally chosen for the ERP experiment are indicated in the rightmost column. A one-way AVOVA on data of set 1 yielded a significant effect of ST $\left(F\left(2,1^{-7}\right)=6.98, p<0.01\right)$. The ratings of set 1 clearly indicate that DA sentences were more often judged as awhward than DL: and At sentences. A post-hoc analysis (Scheffé test) confirmed this result with a minimum significant difference of $9.6 \%$.

With respect to all sentences. a clear ST effect was found $(F(2,357)=16.14 . p<0.001)$. Again, the Scheffé test (critical difference. 6.7\%) revealed that this effect was caused by a significant difference between DA sentences and the two other types of sentences, which in turn did not differ from each other. These results validate the intuition that a garden path may arise from certain object-object ambiguities. However, in comparing the two sets, this effect is somewhat obscured in set 2. All sentence types received overall more awkward responses in set 2 than in set 1 . A twc.way (ST $\times$ Set) ANOVA was carried out that revealed a significant main effect for Set $(F(1,354)=13.84, p<0.001)$. Importantly: there was no ST $\times$ Set interaction $(F(2.354)=1.19 . p=$ 0.3). This indicates that the principal ambiguity effect did not change. The reason for the enhancement of awkward responses in set 2 might simply be that sentences were not as carefully constructed as for set 1 . Note in particular that due to the use of bare NPs, the resulting generic reading may have made them liable to plausibility effects. (Most of them made general statements as to what should or should not be done with respect to certain persons or things.) In order to avoid confounding influences of plausibility judgments in the ERP experi- ment. sentences were selected according to the criterion of having received approximately similar ratings in the D) and AA conditions.

\section{ERP Experiment}

ERPs to the First Word

The first word of the sentences elicited a sequence of ERP components with a topography that is usually asso. ciated with verbal stimuli. A first negativity at about 140 msec $(\mathrm{N} 1)$ is followed by a centro-frontal positivity with a peak latency of about $250 \mathrm{msec}$ (P2). Thereafter a negativity most prominent at central sites $(N+(0)$ shifts into a broad positive wave $(\mathrm{P} 600)$ with a maximum at parietal electrode positions. ERPs of all three sentence conditions appear rather similar (Figure 1). MANOVA 1 for each time epoch considered revealed neither an effect of ST nor an interaction with ES (ST $\times$ ES). Although a slight negative shift in ambiguous nouns within 300 to 450 msec after word onset becomes obvious this effect was of no statistical significance $(F(2.32)=1.96$. $p=0.166)$

\section{ERPs to the Period Betueen Relative Clause and the Final Verb}

Vo effect occurred at the auxiliary immediately after the relative clause. Considering the whole period after the auxiliary no significant potential shift (mean amplitude within 1500 to $4900 \mathrm{msec}$ : $\left.\left(F(2,32)=0.2^{-}, p=0 . .^{-6}\right)\right)$ was found to differentiate between the three sentences trpes (cf. Figure 2). The additional analysis of three independent time windows ( 1500 to $2000 \mathrm{msec}:(F(2.32)=$ $0.28 . p=0 .-4): 3100$ to 3600 msec: $(F(2.32)=0.05 . p=$ $0.94:$ and 4500 to 5000 msec: $(F(2.32)=0.38 . p=0.68)$ did not reveal any effect. No ST $\times$ ES interaction arose in any of the time windows.

\section{ERPs to the Final Verb}

Inspection of the waveforms triggered by the final verbs (Figure 3) reveals a fronto-central pronounced $\mathrm{N} 1$ with

Table 2. Overall percentage of awkwardness ratings for the two sets of sentences of the questionnaire study: The rightmost column (ERP set) indicates the effect for sentences selected for the ERP study. Minimum significant differences were computed according to the Scheffé post-hoc analysis

\begin{tabular}{lcccc}
\hline & Set 1 & Set 2 & All & ERP set \\
\hline Sentence type: & & & & 35.6 \\
Dative/ambiguous & 31.8 & 39.4 & 39.6 \\
Dative/unambiguous & 20.6 & 33.1 & 26.8 & 23.9 \\
Accusative/ambiguous & 18.2 & 22.3 & 20.2 & 19.0 \\
Minimum significant difference & 9.6 & 9.0 & $6 .-$ & \\
\hline
\end{tabular}




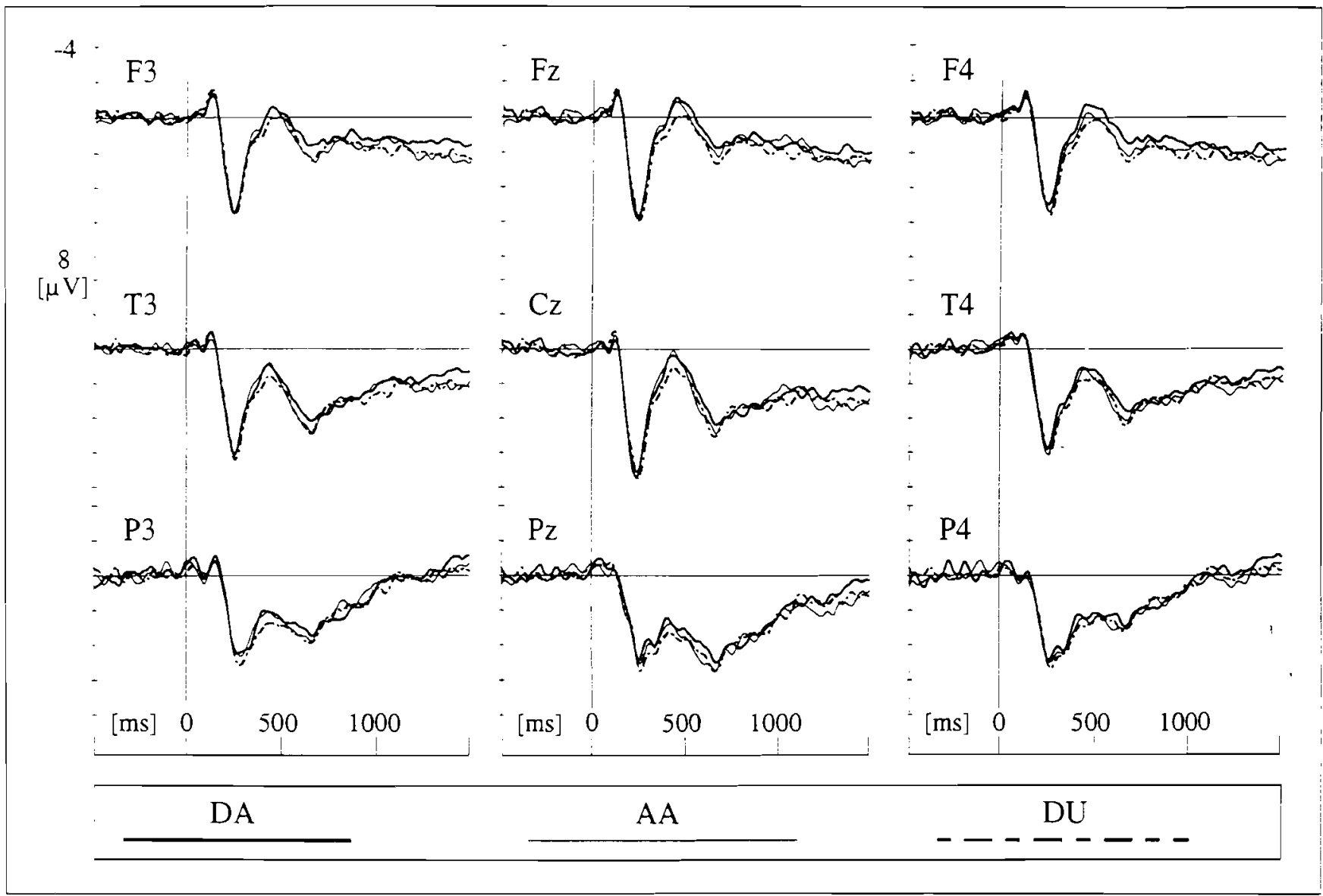

Figure 1. Average ERPs for the first noun. Waveforms for dative ambiguous (DA) accusative ambiguous (Ad). and dative unambiguous (DU) sentences are superimposed.

a mean peak latency of 141 msec after stimulus onset and a subsequent positive deflection with a mean peak latency of 264 msec (P2). The following centro-posterior pronounced negative deflection within about 300 to 600 msec (N400. mean peak-latency: $365 \mathrm{msec}$ ) appears with a sharper negative slope than in the first word of the sentence. The subsequent marked positive wave that is much more prominent than in the initial word of the sentence is a well-known sign of the word's tinal position in a sentence (Friedman. Simson. Ritter. \& Rapin. $\left.19^{-5}\right)$.

V1. This peak was quantified by a mean amplitude within - 0 to 150 msec. HA VOVA I indicated no effect of ST or an ST $\times$ ES interaction but had a significant topography $(F(18.288)=12.9 . p<0.001)$. Peak-latency analysis revealed a main effect for electrode positions ( $F(18.288$ ) $=3.5 . p<0.05)$ mainly due to shorter latencies at frontal electrodes.

$P \geq(150$ to 300 insec). MAVOVA I showed a significant main effect for ST $(F(2.32)=5.42: p<0.05)$ with no further interaction. The analysis of peak latencies reraled an additional effect for topographr $(F(18,288)=$
5.4, $p<0.01$ ) that is mainly caused by an earlier onset at frontal sites

$.1300-450$ (300 to $450 \mathrm{msec}$ ). Starting about $300 \mathrm{msec}$ after word presentation, a clear differentiation of waveforms according to the experimental conditions (ST) becomes visible. A clearly pronounced negative shift for DA sentences contrasts with more positive waveforms for $A A$ and DU sentences that are. in turn. quite similar. Vevertheless, condition $D L$ remains slightly more negative throughout the whole epoch. MANOVA I for the 300to 450 -msec window resulted in a significant ST effect $(F(2.32)=11.45 . p<0.001)$ as well as in an ST $\times$ ES interaction $(F(36,576)=2.64 . p<0.05)$. MANOVA II. however, did not confirm the ST $\times$ ES interaction. Subsequent pairwise comparisons between the sentence tvpes (Table 3) validate the striking difference between $\mathrm{DA}$ and $\mathrm{AA} / \mathrm{DL}$. The small difference between $\mathrm{AA}$ and $\mathrm{DC}$. however, was not significant.

N450-600 (450) to 6000 msec). No peak latencies were computed for this and the following time epochs be cause distinguishable peatis were hard to determine MANOVA I vielded significant effects for ST $(F(2,32)=$ 
Figure 2. Average waveforms withen the recording interial berween the relative clausc and the tinal verb. The trit ERP represents the response to the auxilian?

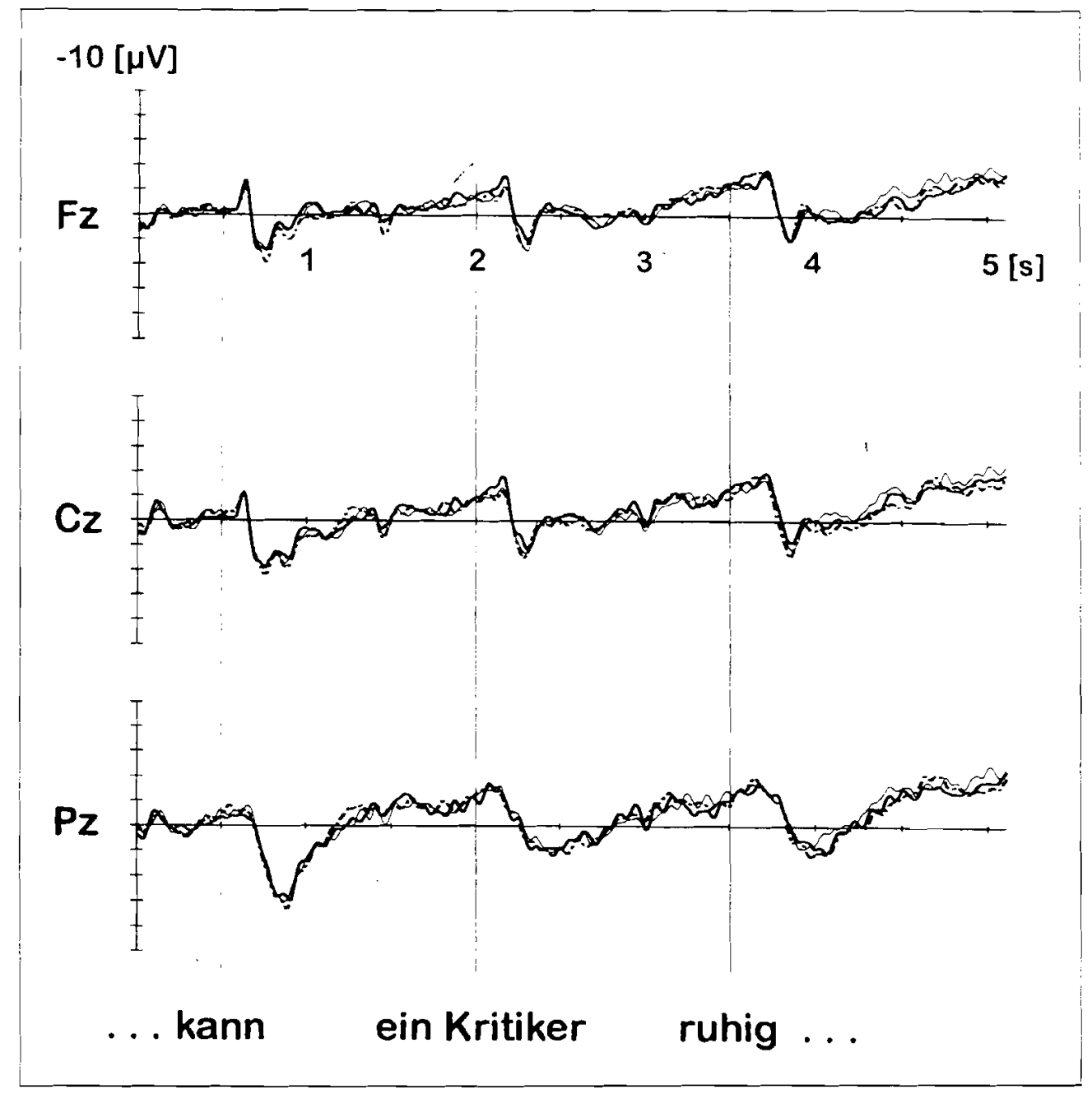

9.59, $p<0.001)$. ST $\times \operatorname{ES}(F(36.576)=4.43, p<0.01)$. and for ES $(F(18.288)=4.32 . p<0.05)$. MANOVA II confirms the ST $\times$ ES interaction $(F(2,32)=2.73, p<$ 0.01 ). indicating marked differences berween $\mathrm{DA}$ and AA/DL for central and right posterior sites. Pairwise comparisons (Table 3) revealed significant differences between DA and $\mathrm{AA}$ and between $\mathrm{DA}$ and $\mathrm{DL}$. whereas no difference was found between AA and DL. Figure 4 demonstrates the results of separate ANOVAs for each elec trode position testing the ST effect.

P600-50 (600 to $750 \mathrm{msec})$. In the overall MANOVA I a significant main effect was found for $\mathrm{ST}(F(2.32)=5.42$ : $p<0.01$ ). As Table 3 indicates, this was because of significantly enhanced negative amplitudes in condition DA vs. AA/DT. MANOVA II revealed a significant ST $\times$ ES interaction $(F(36,576)=2.21 . p<0.05)$. Single-electrode ANOVAs revealed significant ST effects for central and right parieto-occipital regions (Figure 4).

$P^{750-900}$ ( 750 to $900 \mathrm{msec}$ ). Overall main effects were found for ES $(F(18.288)=9.59, p<0.001)$ indicating larger posterior than frontal amplitudes as well as a marginal significance for ST $(F(2.32)=2.55 . p<0.095)$. Interestingly. MANOVA II indicated a significant $\mathrm{ST} \times \mathrm{ES}$ interaction $(F(36.576)=2.02 . p<0.05)$.

Slow Wave (1000 to $1500 \mathrm{msec}$ ). In MANOVA I. ST (F(2. $32)=4.15 . p<0.05)$ approached significance. and in MANOVA II ST $\times$ ES was almost significant, $F(36.576)=$ 2.04, $p=0.058$. Interestingly. in contrast to the four preceding time epochs. the ST effect results from a pronounced difference between condition AA and the two dative conditions. This becomes particularly obvious from the pairwise comparisons shown in Table 3 . The similarit of DL and At has now changed toward a similarity of DA and DU. with both dative conditions yielding more negativity than the accusative condition. Furthermore. subsequent single-electrode AVOVAs testing for the ST effect point to a fronto-central maximum together with a left hemisphere pronounciation (Figure 4)

In order to further evaluate the impact of sentence type on longer-lasting shift processes. difference waves 


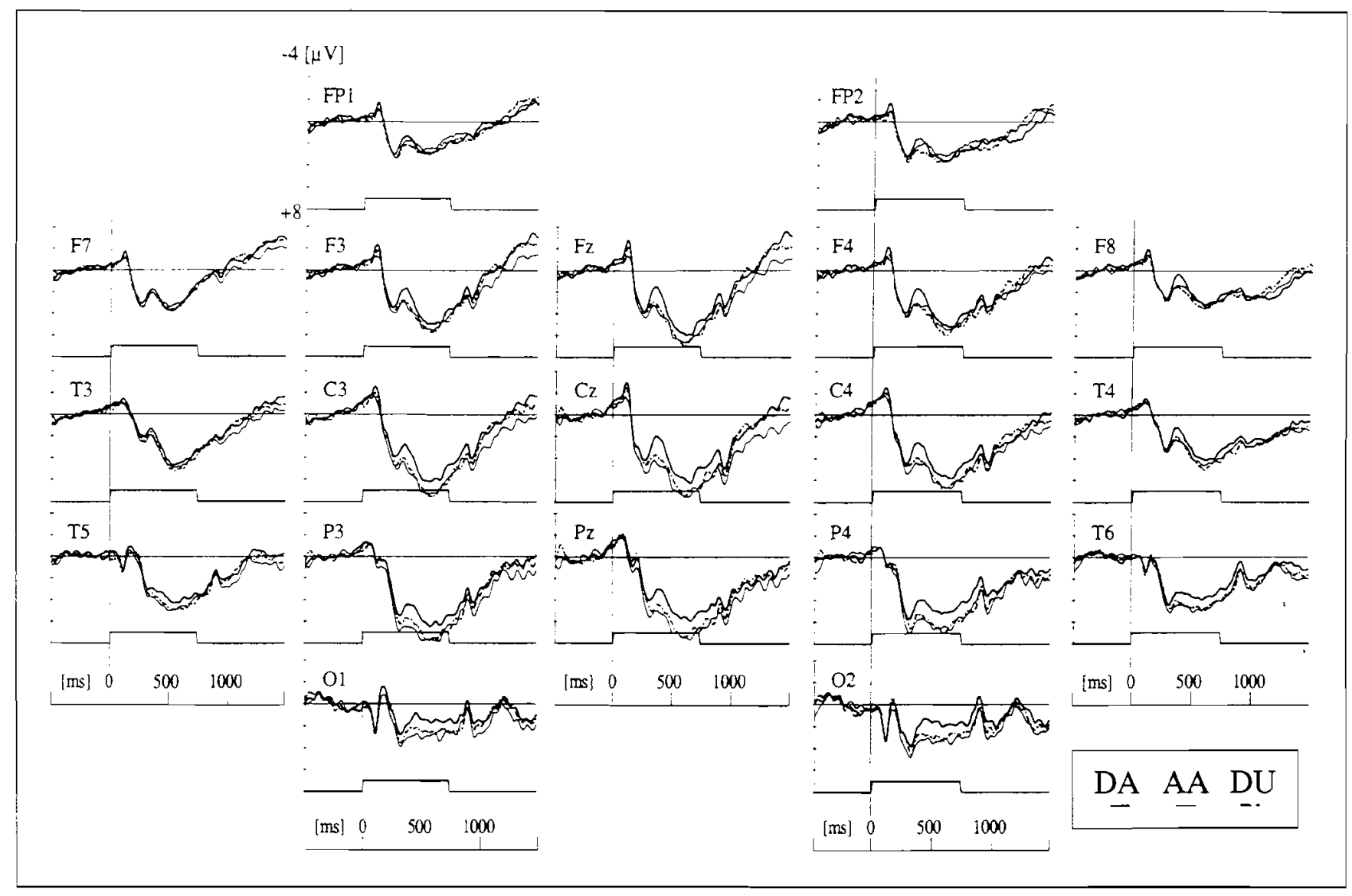

Figure 3. Average ERP waveforms evoled by the tinal verb of dative ambiguous (DA). dative unambiguous (DL). and accusative ambiguous (AA) sentences. The trate at the bottom of each diagram indicates the time window in which the word occurred on the video screen. At about 900 msec a short biphasic potential (N1/Pl complex) with a maximum at occiptal electrodes is visible: it represents the offset potential to the disappearance of the final word

(DU-DA and AA-DA) were computed for an intervall of $2500 \mathrm{msec}$. Figure 5 shows the result for $\mathrm{Cz}$. The time range of about 300 to $900 \mathrm{msec}$ reveals similar positive distances of $D^{\prime}$ and At from DA. which for the following 500 msec change into similar negative distances of DA and $\mathrm{DC}^{-}$from $\mathrm{AA}$. This picture, in turn, changes in the sense of a reoccurrance of the waveform relations within 300 to $900 \mathrm{msec}$ in the time window of about 1400 to $2000 \mathrm{msec}$

\section{Error Rates}

The error rate in recognizing a word that has or has not occurred in the previous sentence can roughly be treated as an index of how accurately the sentence was read. Differences between sentence conditions could indicate some unspecific sources of variance as having an impact on ERPs. However. no such differences were found. The mean total error rate was $2.84(0.91)$ per 120 sentences. A repeated measures ANOVA revealed no ST

Table 3. Sub-ANONAs providing a further analysis of the ST effect for the final verb using pairwise comparisons between the sentence types (1)A is At. DA is. DL, and AA is. DC). Shown are $F$ values $(d f=(1.16)$ ) and corrected Error Type I levels (see "Methods")

\begin{tabular}{|c|c|c|c|c|c|c|}
\hline \multirow{2}{*}{$\frac{\text { Time uindou }}{\$ 3(0)-450}$} & \multicolumn{2}{|c|}{$D A$ ISA AA } & \multicolumn{2}{|c|}{$D A$ is $D C^{r}$} & \multicolumn{2}{|c|}{$A A$ is. $D C^{\circ}$} \\
\hline & 29.62 & $p<0.00 \mathbf{1}^{\prime}$ & 15.20 & $p<0.01$ & 0.23 & $p=0.64$ \\
\hline$x+50-600$ & $13-0$ & $p<0.01$ & 20.02 & $p<0.001$ & 0.09 & $p=0^{--}$ \\
\hline $\mathrm{P}(0)(0)-50$ & 8.93 & $p<0.01$ & $11 .^{-0}$ & $p<0.01$ & 0.09 & $p=0 .^{--}$ \\
\hline $\mathrm{P}^{-5}-50-900$ & 3.29 & $p=0.09$ & 4.39 & $p=0.052$ & $0.1^{-}$ & $p=0.68$ \\
\hline Slow wave &.-+8 & $p<0.05$ & $0.0+$ & $p=0.8+$ & $6.0^{-}$ & $\rho<0.05$ \\
\hline
\end{tabular}




\section{Topography of significant Differences}

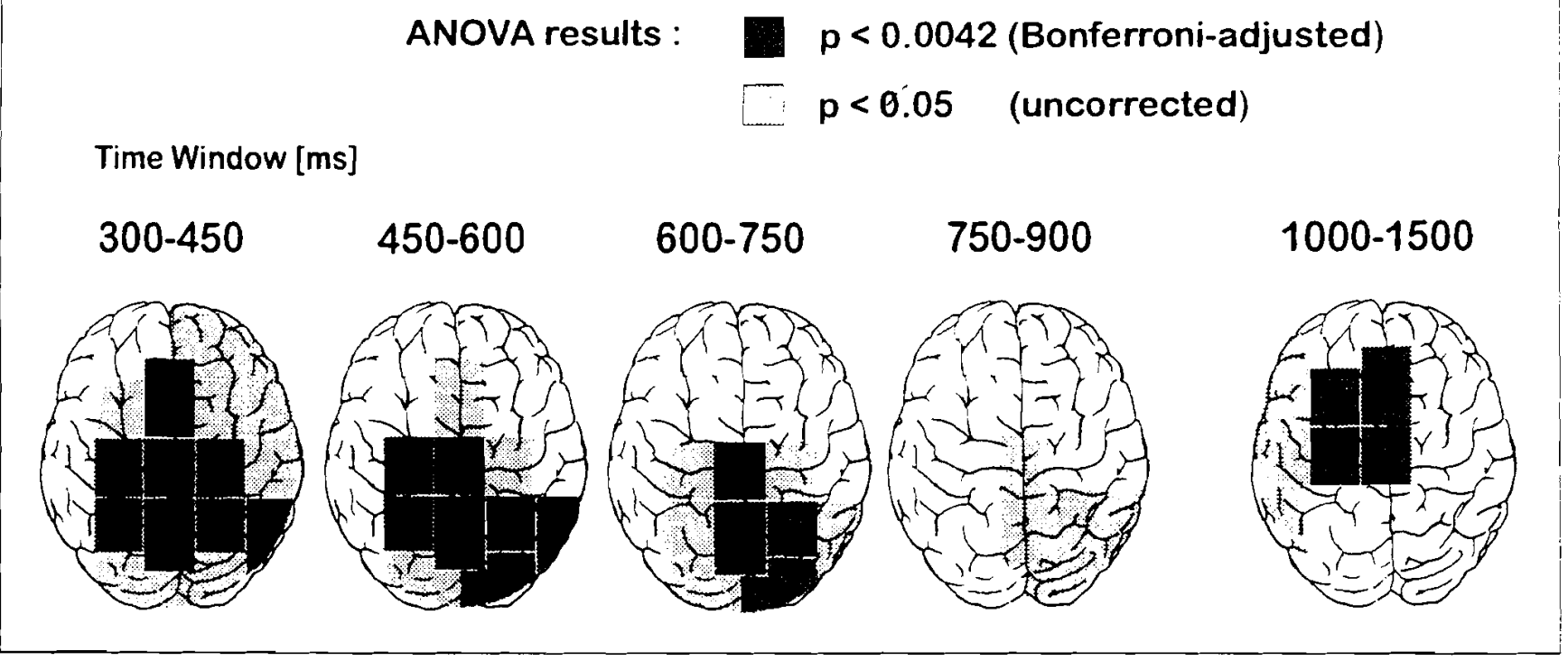

Figure 4. Principal topography of significant differences between the waveforms to final verbs in the three sentence rypes Dart eray squares indicate locations of Bonferroni-adjusted significant waveform differences revealed by single channel ANONAs.

effect $(F(2.32)=2.42, p=0.11)$. However, the factor Experimental Part (PART)-recall that there were two blocks of stimuli-became significant, indicating a slightly rising error rate for the second part (PART $1: 2.4$. PART 2: 3.28).

\section{DISCUSSION}

\section{The Processing of Pure Case Ambiguities}

Results of the questionnaire study clearly confirm the intuition concerning a garden-path effect in processing PCAs of type (8b). Dative ambiguous sentences were more often judged as awkward than both dative unambiguous and accusative ambiguous sentences. In the investigation of ERP waveforms, the most prominent result was a clear centro-posterior pronounced negative shift after final verbs in dative ambiguous (DA) sentences Starting about $300 \mathrm{msec}$ after word onset, the shift begins with a negative enhancement of the $N 400$ and continues until about $900 \mathrm{msec}$ (about the time when the word disappears from the video screen). The topography of this negative shift turns out to be similar to a "classical" N400 topography (Kutas, Van Petten, \& Besson. 1988). In contrast. unambiguous sentences that terminate with a dative verb (DL) and ambiguous sentences with a final accusative-assigning verb (AA) showed more positive and more similar waveforms within this time window:

Our result is consistent with the assumption that case ambiguities are resolved on-line. Certain case options that are morphologically as well as syntactically possible are obviously not considered by the parser. In our materials, the option that the initial ambiguous NP may carr! the dative case is not pursued by the parser, and therefore revisions of the parser's assignments concerning case are necessary as soon as a verb is encountered that requires a dative object. This interpretation is supported by the pattern of ERP responses to the initial ambiguous NP itself. No significant ERP characteristic was found to differentiate between the morphologically marked unambiguous (DC) and the morphologically ambiguous initial nouns (DA, AA). This seems to imply that, at least under the conditions of the present experiment. morphological ambiguity does not have a particular impact on processing at the initial NP and that there might be rather early and fast commitments with respect to case in the sentences used. ${ }^{2}$

The results clearly show that not all possibilities are assigned equal weight by the parser when the caseambiguous NP is processed. More specifically the ambiguous NP is considered to bear the nominative or accusative case. whereas the option of assigning dative case to the initial NP is neglected. However the data do not tell us whether the commitments made by the parser are even more specific. There is ample evidence in the literature on processing German and related languages like Dutch that suggests that an ambiguous initial $\mathrm{NP}$ in a sentence is typically taken to be nominative..$^{.}$If the initial assignment is nominative. it will be discarded as soon as the auxiliary (following the relative clause) is received. The mismatch in agreement features between the plural NP and the singular auxiliary shows that the initial NP cannot be assigned the nominative case. In the 
Figure 5. Difference wave. forms for dative ambiguous dative unambiguous (DA-DT) and darive ambiguous - accusalive ambiguous (DA-AA) forms of the tinal verb

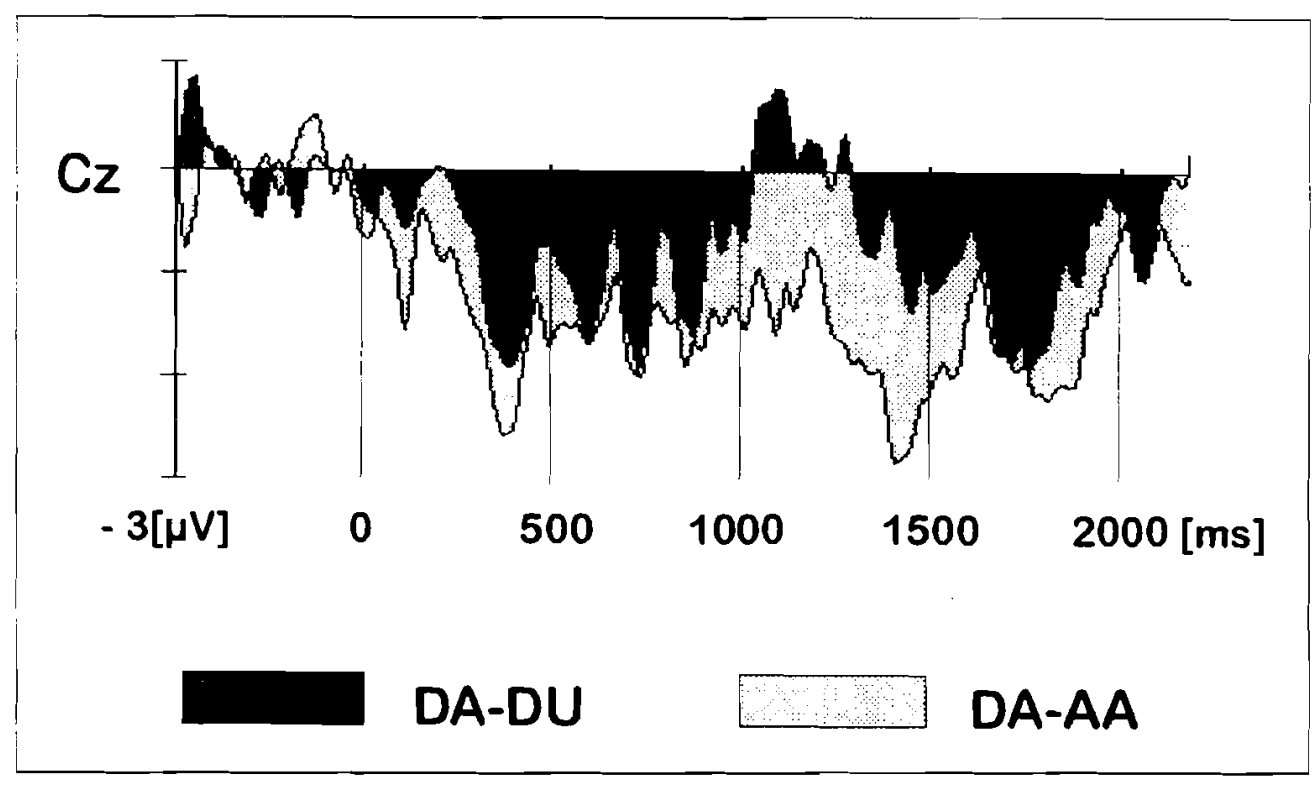

absence of further information. the next choice is to assign the accusative case. Therefore, ERPs to the auxiliary are of interest. As shown by Figure 2. however. no difference in variation of the waveform was found at this point. Given the previous findings, the absence of any disambiguation effect at the auxiliary was unexpected. This may point to a processing difference between the special type of NPs (i.e., generic NPs) used in this experiment and definite NPs or wh-phrases that have been investigated in previous studies. Because this problem was not our primary concern. we will not pursue the issue any further.

With respect to the parser's strategy in dealing with the remaining dative is. accusative ambiguity after the auxiliary, it is of particular interest to consider the long recording period between the auxiliary and the final verb. Recent research (King \& Kutas. 1995) has shown that the processing of sentence regions that strain working memory (object relatives rs. subject relatives) was associated with a frontal negative shift. A frontal positive shift was assumed to indicate the ease of successive integration of items in the sentence due to low memory load (subject-relative sentences). If the dative and accusative object interpretations were both held in working memory: we would in both sentence types expect a sustained frontal negative shift until the final verb. whereas dative unambiguous sentences should trigger a more positive shifting. This was clearly not the case in the sentences under discussion. This speaks against an organization that causes the sentence processor to hold the two case informations in working memory until the disambiguating region is reached. ${ }^{+}$

In sum. the prominent negative shift that was observed in the DA condition but not in the At condition. the lack of a pronounced ambiguity effect at the initial NP. and the lack of a frontal negative shift between the auxiliary and the final verb in ambiguous sentences are most consistent with the assumption that the parser makes an immediate commitment as to the assignment of case to the initial NP. One cannot be sure that a particular case is assigned to the first NP, but one can be sure that the option to assign dative is excluded.

Before we turn to a discussion of the functional significance of the ERP variation in response to PCAs, we will briefly outline some consequences that our findings have for parsing theories. As we have seen in the introduction. there are several ways of capturing the existence of garden-path effects theoretically. but common to all approaches is the assumption that the parser will treat one of the possible continuations as privileged. The precise mechanisms that govern the parser's choice mav vary from theory to theory. In general, however. they rely on the structural alternatives of the phrase that may be pursued in the completion of a sentence at a point of ambiguity. They are designed to ensure that the parser pursues the simplest structural assignment that is compatible with the input.

PCAs are fundamentally different from structural phrases or filler-gap ambiguities. The competing analyses in PCAs do not differ with respect to structural complexity of a phrase. which therefore cannot be appealed to in deriving processing preferences. For the same reasons. the processing difficulty that arises in PCAs if the preferred continuation turns out to be wrong cannot be due to revisions of phrase structure.

We propose that the case assignment decisions of the parser in PCAs are sensitive to the linguistically defined distinction between accusative and dative case. The accusative is a structural. whereas the dative case is a lexical. The main difference between the two types of case amounts to the following: Dative as a lexical case is idiosyncratic and thus needs a specific lexical licenser (e.g. a verb that is subcategorized for a dative NP). Accusative as a structural case is the regular object case. 
It is licensed whenever an VP appears in the grammati cal relation of a direct object. (i.e.. in phrase structure terms as a sister of the verb). A specific lexical licenser is not necessary. ${ }^{3}$ We suggest that the preference of the accusative case over the dative case is due to a general preference for the structural case over the lexical case. as expressed in the generalization in (9):

(9) In the absence of morphological or syntactic evidence to the contrary, prefer structural over lexical case.

The parsing principle in (9) may well derive from the general strategy of the parser to act cautiously (i.e., to make minimal assumptions about the structure of the input). If the parser receives an ambiguous NP that must function as an object, the minimal assumption is that it is an accusative object because accusative case-in contrast to dative case-is not dependent on a specific class of verbs and therefore does not predict the occurence of a lexical licenser later in the input. Consequently; ambiguous object NPs are assumed to carry the accusative case."

To derive the processing difficulty that arises if a case-ambiguous NP is followed by a dative-assigning verb. a similar path may be taken. The information that the ambiguous NP could be assigned dative case seems to have been lost after the parser has made a choice in favor of accusative case. Therefore. the occurrence of a dative-assigning verb forces the parser to check to see whether the object NP may also be able to bear the dative case. To check this, the lexicon where this kind of information is stored has to be consulted. We suggest that the need to leave the proper domain of parsing and to reenter the lexicon is responsible for the processing difficulty associated with PCAs. As will be argued below. this processing account of PCAs also explains why the garden-path effect in our sentences is connected with an enhanced $\mathrm{N} 400$ and not with a late positivity:

\section{Why an N400 and No Late Positivity? ERP Sensitivity to Different Types of Reanalysis}

So far. the discussion of the parsing strategy that governs the processing of PCAs was based on the implicit logic of ERP variation. but not yet on the functional significance of ERP components themselves. As outlined in the introduction. ERP research on syntactic sentence processing revealed two major influences on later ERP components related to the processing of syntactic anomalies. First, positive-going waves (P600. SPS) have been repeatedly found that also seem to accompany syntactic garden paths. These late positivities may be related to processes of reanalysis (Osterhout et al.. 1994) or to the detection of a mismatch between the analysis pursued by the parser and further input (Hagoort et al.. 1993). Secondly: left lateralized negative waves have been reported as indicative of phrase-structure violations
(Friederici et al. 1993: Neville et al. 1991) and subcategorization violations (Rösler. Pütz. Friederici. \& Hahne. 1993). In contrast to these findings, the garden-path effect in sentences of type ( $-b)$ was not related to any of the reported syntactic ERP components and in particular not to a P600 or to an SPS. Rather. a classical $\mathrm{X}+00$ pattern appeared that had the well-known centro-posterior and slightly right topography (Kutas. Van Petten. \& Besson. 1988). This general result may at first sight be surprising. but we believe it can be consistently integrated into a concept of $\mathrm{N} 400$ that goes beyond the functional role of reflecting only semantic expectancy. (Kutas \& Kluender. 1994: Van Petten. 1995).

Late positive components seem to be indicative of reanalysis following a garden path in classical types of phrase structure or filler-gap ambiguities (Mecklinger et al.. 1995: Osterhout et al.. 1994). In PCAs. the initial commitment to structural case (accusative/nominative) triggers a need for reanalysis after the appearance of a dative-selecting rerb. but this is accompanied by a negative potential shift without a late positivity. This suggests that varying processes underlying reanalysis might account for the different ERP effects. Indeed, in almost all studies that investigate syntactic ambiguity; reanalysis was based on the computation of alternative phrase structures or filler-gap dependencies. As suggested by data in Mecklinger et al., the latency and amplitude of the syntactic positivity vary depending on the complexity of the revision operations that manipulate phrase structure relations or filler-gap dependencies. In contrast. the object-object ambiguities presented here are not mirrored by phrase structure alternatives or by filler-gap ambiguities. Reanalysis requires the retrieval of form class information from the lexicon or from the morphological system." Syntactic revision can be performed solely by altering the case assignment to the first NP. and no manipulation of phrase structure or filler-gap dependencies is required. Thus, the lack of phrase structure revisions may plausibly account for the absence of a pronounced late positivity:

Although phrase structure revisions will be computed within the parsing module. reaccessing the lexicon in order to retrieve information about the lexical case goes beyond the parsing module proper. The negativity that starts with an enhanced $\mathrm{N} 400$ could then be indicative of recruiting additional lexical information. This interpretation suggests that syntactic reanalysis following a garden path does not uniformly manifest itself in late positive waves of the ERP. Rather. the particular processes that underlie reanalysis have to be taken into account. and here it appears that different systems of syntactic information used in reanalysis (e.g. phrase structure and morphological information stored in the lexicon), lead to different ERP signatures.

Let us now turn to the question of why an $\mathrm{N} 400$ should arise in reanalysis as it occurs in the processing of PCAs. The N 400 seems to be not exclusively related 
to a sentence-level semantic expectancy in the sense of Kutas and Hillyard's (1980, 1984) earlier reasoning. Halgren $(1990)$ considers . N400 to be tied to lexical encoding and integration within a cognitive context, engaging remote semantic memory as well as primary memory. He considers the ease of lexical integration to be inversely related to $\mathrm{N} 400$ amplitude modulation. Evidence from masked priming (Brown \& Hagoort, 1993) or priming while applying different levels of representation (Chwilla, Brown, \& Hagoort. 1995) support the view that $\mathrm{N} 400$ is sensitive to processes of lexical integration. Thus, our results imply that the N400-component should be seen as a shift that corresponds to the additional lexical activation and integration that is needed for the release and reinterpretation of case-related information. An earlier study of German (Münte \& Heinze. 1994) shows that semantically appropriate final nouns in a sentence with inappropriate case morphology ( ${ }^{*}$ Der Zollbeamte kontrolliert den Koffer-s, "The customs officer checks the x. suitcase GEX) also elicit an N400-like component. One can conclude from these data that disconfirmed expectation of a certain case morphology acts as a source of N400 modulation. Semantic expectations are satisfied. but the aberrant case morphology renders the sentence syntactically unacceptable. The negative component might therefore reflect semantic as well as morphological expectations. both of which are rooted in a particular lexical choice.

\section{Possible Problems}

We will now briefly discuss some effects that might have confounded the present results. First, one could argue. as Hagoort et al. (1993) do. that the N400 occurs right after the parsing problem. which would suggest an immediate relationship between syntactic violation and the downstream conflicts for semantic integration that would be needed to reach a coherent message level of sentence representation (Osterhout \& Holcomb. 1995). However. in our experiment DA sentences did not vield any semantic implausibility resulting from the disambiguating word. as was the case for implausible assignments in the Hagoort et al. study and in a study of Garnsey: Tanenhaus. and Chapman (1989). In addition. hoth DA and DL sentences imposed comparable semantic constraints on the choice of the final verb. DA and At sentences were identical up to the final verb. Thus. $\mathrm{N}+00$ enhancement in DA sentences cannot be caused by differences with respect to semantic expectations. One closely related objection could stress the fact that disimbiguation might be confounded with the final position of the sentence. where sentence wrap-up. decision. and response processes take place (Osterhout. 199-1). Sentences containing a syntactic xiolation elicited an $\mathrm{V} f(0)$ to the final word that was not anomalous itself (osterhout \& Holcomb. 1992: Osterhout \& Mobley. 1995). and syntactic violations embedded in a sentence caused a $\mathrm{P} 600$ response. whereas the same violation elicited an $\mathrm{N} 400$ at final position of the sentence (Osterhout. 1994). However, in the present experiment. no syntactic violation occurred at any position in the sentence that would have necessitated the semantic interpretation of an ungrammatical sentence. The three sentence types used were equivalent with respect to grammatical well-formedness. Response preparation or decision making can hardly have had a confounding influence because the subsequent word-recognition task was entirely superimposed: no predictions for a certain response could be drawn from it.

Another possible artifact could have been due to different occurrence frequencies of dative- versus accusative-selecting verbs. Word frequency has been shown to bear an influence on $\mathrm{N} 400$ and later positive components of the ERP (Rugg, 1990; Van Petten \& Kutas, 1990), such that less frequent words were associated with larger N400s. Thus, the overall smaller number and therefore less-frequent occurrence of dative-selecting verbs in German could. in principle, have caused the confounding effect of a larger $\mathrm{N} 400$. This line of reasoning is not convincing. however, because in this case no difference between DA and DU sentences would be predicted. Notice also that the head nouns could equally well appear in accusative and in dative case. Furthermore, investigations of the interaction between sentence context and word frequency (Van Petten \& Kutas 1990. 1991 ) have found that word frequency only influences the initial but not an intermediate or final open class word of a sentence. Thus. word-frequency effects are eliminated rather early in a meaningful sentence (Van Petten, 1995). Nevertheless. in contrast to the accusative ambiguous (AA) sentences, a slight but nonsignificant negative shift for dative unambiguous (DA) sentences was found. We can therefore not rule out minimal influences of global case-related word-form frequency. Finally: the repetition of a word in a comparable sentence context has been shown to attenuate the N400 and to enhance the positivity thereafter (Besson. Kutas. \& Van Petten. 1992; Besson \& Kutas. 1993). Although word repetition occurs throughout the two experimental runs. it is unlikely to be reflected by our ERP data because somewhat larger repetition ratios for dativeassigning verbs would be expected to cause N400 attenuation ( $\mathrm{P} 600$ pronunciation) rather than enlarged $\mathrm{V}+00 \mathrm{~s}$ in DA sentences in our experiment.

\section{Late Effects: The Slow Wave 1000 to $1500 \mathrm{msec}$ (Final Verb)}

Beginning about 1000 msec after the presentation of the final word, topography and amplitudes of the ERPs for the three sentence types change into different patterns. Similarly enhanced negative shifts can now be found in DA as well as in DL sentences. AA sentences remain significantly more positive (cf. Figure 5) 
In contrast to the previous time window $(300$ to 1000 msec). amplitude differences are most striking at centroanterior sites with a leftward astmmetry (Figure f). Left anterior negativities (LAN) have been shown to be indicative of working memory load during the parsing of unbounded dependencies (Kluender \& Kutas. 1993: Neville et al., 1991). It is unlikely that the present left anterior negative shift within a considerable later time epoch represents such a parsing-associated process Rather it could be associated with a subsequent interpretive process related to differences between the accusative and dative case. Various experimental conditions employing working memory processes have been found to evoke late anterior negative shifts. Lang et al. (198-) demonstrated a frontal slow negative potential shift in a verbal concept formation task when previous experience becomes increasingly integrated in the process of new concept formation. In an attempt to categorize slow waves of the ERP, it has been supposed (Ruchkin. Johnson. Mahaffey; \& Sutton, 1988) that negative slow waves should be associated with memory scanning and mental imagery. Furthermore, the "frontal aspect" of slow waves seems to be related to the storage and retention of information in short-term memory (Ruchkin, Johnson. Canoune, \& Ritter, 1990). The late negative waves in the current experiment "float" on the initial part of a large contingent negative variation. representing the preparatory activation that precedes the final word-recognition task. It could be argued that memorization activities (presumably in association with a rehearsal of the whole sentence) are superimposed on the general expectancyrelated processes of preparation in order to cope with the recognition task. In fact, data from a phonological working memory task imply that late negative frontal shifts may be related to the phonological rehearsal loop (Ruchkin, Johnson. Grafman, Canoune. \& Ritter, 1992). Provided that this conjecture can be upheld, why should we find memorization of the dative sentences more demanding than memorization of the accusative sentences? Because the present experiment was not designed to control for memorization strategies, any answer would be premature at the present stage of our research. The current data cannot sufficiently address the functional role of waveform variations in this late time epoch: this therefore remains a topic for future research.

\section{CONCLUSION}

The results of the present ERP study imply that the sentence parser makes an early decision to assign case to a case-ambiguous noun phrase instead of waiting for disambiguating information. The parser's choice seems to be governed by a principle that gives priority to the assignment of structural case (nominative or accusative). PCAs are thus immediately resolved. Case-ambiguous and case-unambiguous sentences were characterized by comparable slow potential shifts between the relative clause and the final verb. This is in line with the idea that as a direct consequence of sach early commitment, the parser reduces working memory load by discarding the alternative continuation according to which the NP would carry the dative case. The main evidence comes from the observation that dative-selecting verbs in 10 cally ambiguous sentences elicited a prominent centroposterior negative shift. Time range and topography qualify this negativity as an enhanced $\mathrm{N} 400$ component. Although they elicited more positivity in general. accusative ambiguous (AA) and dative unambiguous (DL) sentences did not differ from each other. We therefore conjecture that this negativity signals a garden-path effect that only arises when the initial case assignment turns out to be grammatically untenable. The novel as pect in comparison with other investigations of ERPs to syntactic ambiguity resolution is that PCAs have the exceptional property of not being mirrored by alternative phrase structures or filler-gap dependencies. The observed negativity selectively reflects reanalysis that is based on a second access of formal lexical information. This interpretation fits well into the concept of lexical activation/integration as the functional side of the $\mathrm{N} 400$ component. The absence of a late positive wave (P600. SPS) indicates that this component is not a general indicator of revision operations to cure the garden path Rather it seems to be confined to revisions that affect the phrase structure representation. The importance of this result is that it reveals a rather nontrivial isomorphy between distinct linguistically defined processes and their neurobiological correlates. If further research along these lines proves it to be tenable, we have additional evidence that the grammar and the parser employ operations that can be distinguished in the functional architecture but also by virtue of the brain's responses.

\section{METHODS}

\section{Questionnaire Experiment}

\section{Subjects}

A total of 120 subjects participated in the experiment All subjects were students at the University of Jena and native speakers of German.

\section{Materials}

A total of 90 sentence triplets were constructed (see Table $1.8 \mathrm{a}$. b. \& c. for a triplet of examples). Two sentences of each triplet started with a case-ambiguous NP. which always was the unique (accusative or dative) object of the clause. One locally ambiguous sentence terminated in a verb that requires an accusative object (8a). whereas the other one terminated in a verb that requires a dative object $(8 \mathrm{~b})$. The third sentence in each 
triplet started with an unambiguously dative-marked noun and terminated in a verb that requires a dative object $(8 \mathrm{c}) .{ }^{8}$ All sentences were constructed according to the schema shown in $(10)$ :

(10) noun relative case finite verb subject adverbial(s) nonfinite verb

Each sentence started with a plural noun that was accompanied by a relative clause. This relative clause was followed by the finite verb that always was an auxiliary or modal verb. The finite verb in turn was followed by the subject. The subject was always a singular NP, and because subject and verb agree with respect to number and person in German, the verb had to be morphologically marked as singular, too. As a consequence. it could be determined at the position of the finite verb that the initial NP could not be the subject and could thus not carry the nominative case. The following NP always turned out to be the subject. This was followed by one or two adverbials that modify the proposition and make the sentence semantically and pragmatically coherent. Each sentence ended with an open-class nonfinite verb. This verb dictated which case was required for the object NP. The final verb of the sentence thus unambiguously determined the case of the sentence's initial object NP. All initial nouns in the sentence were bare plural nouns that fell into two categories. Nouns of the first category were case ambiguous. A noun like Dirigenten (see 8 a. b) can be either nominative:accusative. or dative (under rare circumstances even genitive. but no such condition was involved). Nouns of the second category were items such as Husikern (see 8c). Through the attachment of the morpheme $n$, these were unambiguously marked as datives. If a noun like this heads an NP. this VP can only serve as a dative object. In both nominative and accusative cases. the correct form would be husiker:

The 90 sentence triplets were divided into two sets of +5 sentence triplets each. Set 1 contained triplets 1 through +5 : set 2 contained triplets 46 through 90 . From each set. three lists were created so that each list contained an equal number of sentences in each condition. but no more than one version of any sentence appeared in a list. Each list of experimental sentences was embedded in a list of 39 filler sentences of a variety of sentence types.

\section{Procedure}

The subjects were instructed to read the sentences for comprehension and to judge for each sentence whether it was "natural" or "awhward." No time pressure was imposed. subjects were encouraged to read at their normal speed and to indicate the intuitive impression that ealch sentence made on them. On average the experiment took 20 min to complete.

\section{ERP Experiment}

\section{Subjects}

Eighteen right-handed university students ( 7 males, 11 females) with a mean age of 23.45 (3.71) years took part in two experiments. Handedness was evaluated according to self-report. All subjects were native speakers of German and had normal or corrected to normal vision. Subjects were paid $10 \mathrm{DM}$ per hour for participation. One subject was not included in the final analysis because of massive artifacts.

\section{Procedure}

A total of 180 sentences (i.e., 60 sentences for each case condition), were selected from the stimuli that had been used in the questionnaire experiment. Sentences that gave rise to plausibility judgement effects in the questionnaire experiment (see the "Results" section) were excluded. The final selection was based on the criterion of sentence triplets having similar awkwardness ratings for DU and AA sentences. The total amount of stimulus material was partitioned into three lists. Each list contained 20 sentences per condition ( 60 sentences) that did not occur in other lists. In addition, each sentence of one triplet occurred in a different list. Sentence types (DA, DU, AA) were presented in random order. In order to limit repetitions of the initial sentence parts, subjects performed on two different lists (120 sentences) that were assigned in random combination to all subjects. Hence, the final analysis of average waveforms was based on $66 \%$ within-subjects comparisons and $33 \%$ betweensubjects comparisons for each sentence type. The presentation of each list took about $20 \mathrm{~min}$. The experimental runs were followed by resting periods of about 5 to $10 \mathrm{~min}$. A short $(-\mathrm{min}$ ) unrelated experiment testing word length and nonsense string length effects occurred between the two experiments (data not reported here). The words presented in this experiment were carefully chosen so that they did not interfere with the sentence material.

Sentences were visually presented in chunks as shown in Figure 6 in the center of a 20 -in video monitor. The frames appeared at a moderate time course that proved to be comfortable and guaranteed exact reading. After the presentation of the first word for $1400 \mathrm{msec}$, the entire relative clause occurred for $2800 \mathrm{msec}$. Thereafter the remaining parts of the sentence were presented one word per frame for $750 \mathrm{msec}$. Intervals between sentence parts lasted $750 \mathrm{msec}$. In order to avoid response preparation triggered by the final word as well as to guarantee careful reading of the sentence. an additional word was displayed $2000 \mathrm{msec}$ after the final word. By pressing a mouse button. subjects had to decide whether this word had occurred in the previous sentence or not. The end of each trial was signaled by three asterisks. 


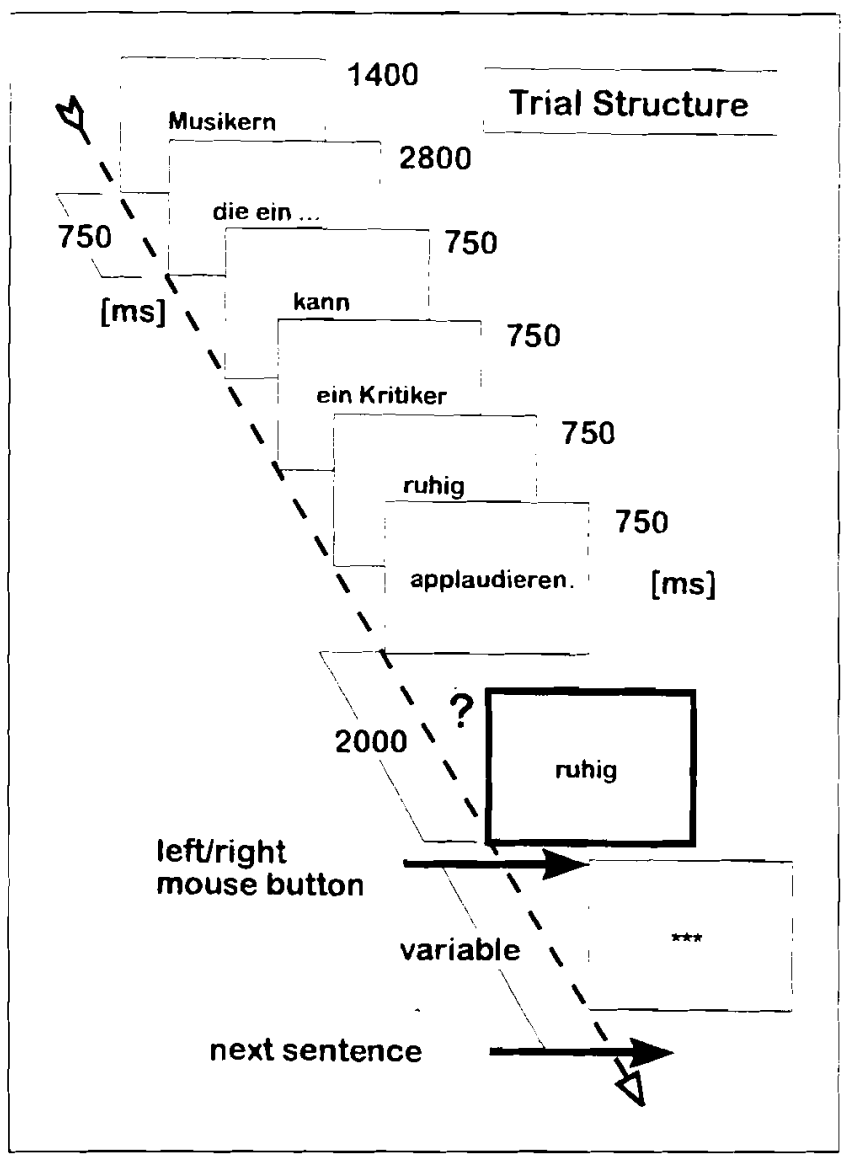

Figure 6. Schematic illustration of the course of sentence presentation in the ERP experiment.

\section{Data Recording}

The EEG was recorded from 19 scalp electrodes ( $\mathrm{Ag} / \mathrm{AgCl})$ referred to linked mastoids according to the International 10-20 system (Fpl. Fp2, F3. F4, C3. C4. P3. P4. O1. O2. F7 , F8. T3. T4. T5, T6. Fz. Cz. Pz: see Jasper. 1958). Horizontal eve movements were monitored by electrodes at the outer canthi of the eyes. and vertical movements were controlled by electrodes localized above and below the orbital ridge of the left eve. Electrodes were placed by a scratch-technique (Bauer. Korunka, \& Leodolter. 1989) in order to eliminate skin potentials. The resistance was typically 5 to $7.5 \mathrm{k} \Omega$ and always less than $10 \mathrm{k} \Omega$. EEG was amplified by a 32-channel device (PDA 989, MES) with a system bandpass (dc to $30 \mathrm{~Hz}$ ) and a digitizing rate of $10 \mathrm{msec}$ per sample for a total sampling interval of $5120 \mathrm{msec}$. Off-line artifact correction was performed using a combination of artifact correction based on a method of Semlitsch. Anderer. Schuster. and Presslich (1986), implemented in the recording software SCAN 3.0 (Neuroscan. Inc., LSA) and a subsequent rejection of insufficiently corrected trials.

\section{Data Anallsis}

ERPs were analyzed separately for the first noun, the last verb, and the epoch between relative clause and final verb. The in toto presentation of the relative clause led to considerabie eve-movement artifacts. Therefore, no analysis was performed for this sentence part. Baseline correction was performed by means of a 500 -msec prestimulus interval. Averaged ERP waveforms were computed for each subject and each sentence type. After the inspection of the grand mean waveforms. time windows encompassing ERP components of interest were determined to calculate mean amplitudes. $v 1$ was measured between 70 and 150 msec. and P2 was measured between 150 and $300 \mathrm{msec}$. For the time range that followed, beginning $300 \mathrm{msec}$ after stimulus onset, average voltage amplitudes were computed for four consecutive 150-msec time intervals. The first two epochs $(300$ to $600 \mathrm{msec}$ ) covered the $\mathrm{N} 400$. and the last two epoches corresponded to the $\mathrm{P} 600$ component. A last epoch (1000 to $1500 \mathrm{msec}$ ) was analyzed for a late slow wave.

Separate statistical analyses were conducted for each time window: In order to determine principal influences of the experimental conditions. Overall (Electrode Positions $\times$ Sentence Type) MANOVAs (MANOVA I) with repeated measures for both factors were performed (General Linear Model implemented in SAS, SAS Institute Inc.). To evaluate true interactions of the sentence type (ST) effects with topographical distributions. additional overall MANOVAs (MANOVA II) were based on a 19-dimensional (electrodes) space representation of the ST effect (McCarthy \& Wood, 1985). In this representation. direction cosines of the ST vectors are independent of differences in vector lengths (ST main effects): that is. the direction cosines are not confounded with spurious topographical effects due to strength differences of a single source. Violations of variance homogeneity were controlled by adjusting dimensions of freedom according to the Huynh-Feldt-Epsilon method (Huynh \& Feldt. 1976). Statistical results of this study are reported with initial degrees of freedom in conjunction with corrected nominal Error Type I levels. To control the enhanced likelihood of Error Type I for the single-electrode ANOVAs, Bonferroni-correction was applied to the nominal alpha-levels.

\section{Acknowledgments}

This study was supported by the Gesellschaft der Freunde und Förderer der Friedrich-Schilier-Lniversität Jena c.V.

Reprint requests should be sent to Jens-Max Hopf. Clinic fisr Veurophysiology, Leipziger Strasse 44. D-39120 Magdeburg. Germany: or via e-mail: ijh@rz.uni-jena.de, or max@marzen. ucdavis.edu 


\section{Notes}

1. Within the Minimalist Program (Chomsky: 1995). accusative and dative objects mav be assumed to occupy different positions in the phrase marker at some abstract level of syntactic representation. Overtly such different positioning cannot be obseried. Because the consequences of such a theory for German remain to be worked out. we will not pursue a reinterpretation of what we have to say here in terms of abstract phrase structure (see also Chomsky, 1995.p. $349 \mathrm{ff}$ for serious caveats concerning checking for case agreement).

2. As indicated above the $\mathrm{N} 400$ component following ambiguous NPs is slightly more negative compared to unambiguous XPs (see Figure 1). Although the effect failed to reach significance. we cannot rule out that the investigation of a larger set of sentences would reveal processing differences between morphologically ambiguous and unambiguous nouns.

3. See Bayer \& Marslen-Wilson (1993) and Bader (1994a) for German. as well as Frazier (1987) for Dutch.

4 . It remains an interesting question for future research to determine to what extent the distance or the nature of constituents between ambiguity and disambiguation influence the parser s decision to discard an alternative case interpretation. 5. Prominent aspects of this distinction can be seen in the fact that the accusative object of a verb promotes to nominative in passives and to (structural) genitive in nominalizations, whereas a dative object never does.

\section{(i) a. ACTNE}

Der General wollte den Rückzugacc aufschieben the general wanted the retreat postpone

"The general wanted to postpone the retreat

b. PASSIVE

Der Rückzugvoy wurde aufgeschohen

c. NOMINALIZATION

Der Aufschub des Rückzugs $s_{\text {(iE }}$

(ii) a. ACTNE

Der General wollte dem Leutnant $\mathrm{DAT}_{\mathrm{D} T}$ helfen the general wanted the lieutenant help

"The general wanted to help the lieutenant"

b. PASSIVE

*Der Leutnant von wurde geholfen

c. NOMINALIZATION

*Die Hilfe des Leutnants $s_{\mathrm{GE}}$

6. Alternatively the preference of accusative over dative could simply be due to a strategy. according to which the parser prefers more frequent to less frequent structures. However. there are no corpora data available on German that would allow this hypothesis to be tested.

- For reasons of space. we cannot address the question of where exactly morphological case information should be located in the grammar

8. In contrast to dative plural NPs. there are no plural NPs that are unambiguously marked for accusative case. A plural accusative. $\mathrm{NP}$ is always ambiguous, at least between the accusative and nominative case. We therefore could not include unambiguous accusative sentences.

\section{REFERENCES}

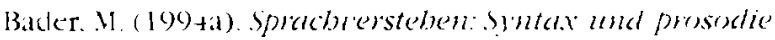
beim lesen /language processing: syntax and prosody in reading: I npublished doctoral clissertation. I niversity of siutgart. sturtgart.
Bader M. (1994́b). Syntactic-function ambiguities. Folia Linguistica. 28. 5-66.

Bauer, H., Korunka, C. \& Leodolter, M. (1989). Technical requirements for high-qualiry scalp DC-recordings. Electroencephalography and Clinical Neuropbysiology, 72, $545-54$ ?

Baver. J., \& Marslen-Wilson, W. (1993). Configurationality in the light of language comprebension: The order of arguments in German. Unpublished manuscript. University of Vienna \& Birkbeck Coliege, London.

Besson, M. \& Kutas, M. (1993). The many facets of repetition: A cued-recall and event-related potential analysis of repeat ing in same versus different sentence contexts. Journal of Experimental Psycbology: Learning, Memory: and Cognition, 19. 1115-1133.

Besson. M. Kutas, M., \& Van Petten, C. (1992). An event-related potential (ERP) analysis of semantic congruity and repetition effects in sentences. Journal of Cognitive Neuroscience, 4, 132 148 .

Brown. C. \& Hagoort. P. (1993). The processing nature of the N400: Evidence from masked priming. Journal of Cognitive Neuroscience, 5. 34-44.

Chomsky. N. (1995). The minimalist program. Cambridge. MA: MIT Press.

Chwilla. D. J.. Brown, C. \& Hagoort, P. (1995). The N400 as a function of the level of processing. Psychopbysiology, 32, 274-285.

Frazier. L. (1978). On comprebending sentences: Syntactic parsing strategies. Unpublished doctoral dissertation. Liniversity of Connecticut.

Frazier. L. (1987). Syntactic processing: Evidence from Dutch. Vatural Language and Linguistic Theory; 5, 519-560.

Frazier. L.. \& Rayner, K. (1982). Making and correcting errors during sentence comprehension: Eve movements in the analysis of structurally ambiguous sentences. Cognitive Psycbolog1: 14, 178-210.

Friederici. A. D. (1995). The time course of syntactic activation during language processing: A model based on neuropsychological and neurophysiological data. Brain and Language. 50. 259-281

Friederici, A. D. \& Mecklinger. A. (1996). Syntactic parsing as revealed by brain responses: First-pass and second-pass parsing processes. Journal of Psycholinguistic Research. 25. $15^{-}-1-6$.

Friederici, A. D. Pfeifer. E. \& Hahne, A. (1993). Event-related brain potentials during natural speech processing: Effects of semantic. morphological and syntactic violations. Cognitice Brain Research. 1. 183-192.

Friedman, D. Simson. R. Ritter. W., \& Rapin. I. (1975). The late positive component (P300) and information processing in sentences. Electroencephalograpby and Clinical Veurophysiologi: 38. 225-262.

Garnsey. S. M. Tanenhaus. M. K.. \& Chapman. R. M. (1989) Evoked potentials and the study of sentence comprehension. Journal of Psycholinguistic Research. 18. 51 60 .

Gibson. E. (1991). A computational theory of buman linguistic processing. Lnpublished doctoral dissertation. Carnegie Mellon Lniversity: Pittsburgh. PA.

Gorrell. P. (198-). Studies of buman sentence processing: Ranked parallel iersis serial models. Lnpublished doc toral dissertation. Lniversicy of Connecticut.

Hagoort. P. Brown. C. \& Groothusen. J. (1993). The syntactic positive shift (SPS) as an ERP-measure of syntactic processing. Language and Cognitile Processes. 8, 439-483.

Halgren. E. (1990). Insights from evoked potentials into the neuropsichological mechanisms of reading. In A. B. 